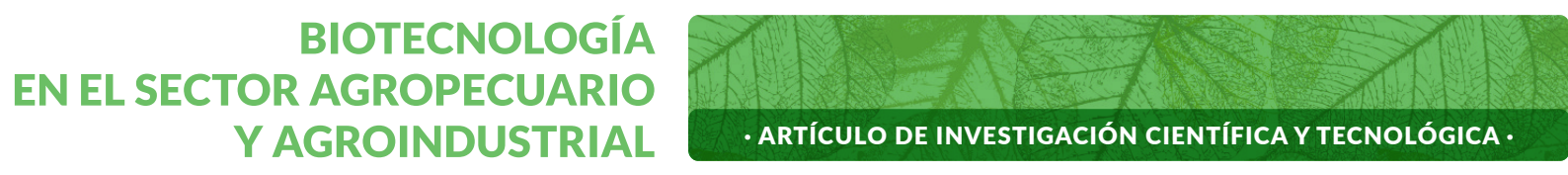

Vol. 16 No 2 · Julio - Diciembre 2018 • ISSN - 1692-3561 · ISSN-e 1909-9959 · doi: http://dx.doi.org/10.18684/bsaa.v16n2.98

\title{
Desvinculación económica del sector extractivo en Colombia
}

\section{Economic decoupling of the extractive sector in Colombia}

\section{Desvinculação econômica do setor extrativo na Colômbia}

\author{
JULIANA SARMIENTO-CASTILLOํ, MARIO PÉREZ-RINCÓN², \\ MAURICIO GÓMEZ-SÁNCHEZ ${ }^{3}$
}

\section{RESUMEN}

Este artículo tiene como objetivo aportar argumentos alrededor de los supuestos beneficios económicos que el modelo extractivista le ha dejado al país, después de más de dos décadas de ejecución en las que ha sido catalizador de pasivos sociales y ambientales, un tema de gran trascendencia académica y política para Colombia. Para tal fin se plantearon dos modelos logarítmicos, sometidos previamente a un análisis de cointegración que garantizó la obtención de regresiones no espurias. Los resultados del primer modelo econométrico arrojaron evidencias de la desvinculación entre

Recibido para evaluación: 6 de Diciembre de 2016.

Aprobado para publicación: 31 de Mayo de 2017.

1 Universidad del Cauca, Facultad de Ciencias Contables, Económicas y Administrativas, Departamento de Economía, Grupo de Investigación Entropía. $\mathrm{PhD}$ (c) en Ciencias Ambientales. Popayán, Colombia.

2 Universidad del Valle, Facultad de Ingeniería, Instituto CINARA, Grupo de Investigación en Comunidad, Ambiente y Sustentabilidad. PhD en Ciencias Ambientales con Énfasis en Economía Ecológica y Gestión Ambiental. Cali, Colombia.

3 Universidad del Cauca, Facultad de Ciencias Contables, Económicas y Administrativas, Departamento de Economía, Grupo de Investigación Entropía. Magíster en Economía Aplicada. Popayán, Colombia.

Correspondencia: jisarmiento@unicauca.edu.co 
el crecimiento económico del país y variables macroeconómicas del sector extractivo, tales como la inversión extranjera directa, el índice de precios internacionales y exportaciones de productos minero-energéticos. Los hallazgos del segundo modelo confirmaron la relación directa entre las exportaciones extractivas y el índice de precios internacionales de minerales e hidrocarburos, así como el comportamiento del PIB de economías emergentes y en vías de desarrollo, que demandan commodities colombianas, no obstante la relación entre estas variables no resultó tan fuerte. Es así como se rechaza la hipótesis de los efectos positivos asociados a la promoción de un modelo de desarrollo basado en la explotación masiva de recursos naturales agotables.

\section{ABSTRACT}

This article aims to provide arguments about the supposed economic benefits that the extractivist model has left to the country, after more than two decades of execution in which it has been a catalyst of social and environmental conflicts, a subject of academic and policy importance for Colombia. For this purpose two logarithmic models were submitted, previously submitted to a cointegration analysis that guaranteed non-spurious regressions. The results of the first econometric model showed evidence of the decoupling between the economic growth of the country and macroeconomic variables of the extractive sector, such as foreign direct investment, international price index and exports of mining-energy products. The discoveries of the second model confirmed the direct relationship between extractive exports and the international mineral and hydrocarbon price index, as well as the GDP behavior of emerging and developing economies that demand Colombian commodities, despite the relationship between these variables was not so strong. Thus, the hypothesis of the positive effects associated with the promotion of a development model based on the massive exploitation of exhaustible natural resources is rejected.

\section{RESUMO}

Este artigo tem como objetivo fornecer argumentos sobre os benefícios económicos suposto que o modelo extrativista deixou o país depois de mais de duas décadas de execução, que tem sido um catalisador para os passivos sociais e ambientais, uma questão de grande importância acadêmica e política para a Colômbia. Para este efeito, dois modelos logit, previamente submetidos a uma análise de cointegração que garantiu regressões não espúrias. Os resultados do primeiro modelo econométrico evidenciaram a dissociação entre o crescimento econômico do país e as variáveis macroeconômicas do setor extrativista, como o investimento estrangeiro direto, o índice de preços internacionais e as exportações de produtos de mineração. Os achados do segundo modelo confirmaram a relação direta entre as exportações extrativas eo índice internacional de preços de minerais e hidrocarbonetos, bem como o comportamento do PIB das economias emergentes e em desenvolvimento que demandam commodities colombianas, apesar da relação entre essas variáveis não ser tão forte. Assim, é rejeitada a hipótese dos efeitos positivos associados à promoção de um modelo de desenvolvimento baseado na exploração massiva de recursos naturais exaustivos.

\section{PALABRAS CLAVE:}

Commodities, Crecimiento económico, Desindustrialización, Extractivismo, Modelos

logarítmicos.

\section{KEYWORDS:}

Commodities, Deindustrialization, Economic growth, Extractivism, Logarithmic models.

\section{PALAVRAS-CHAVE:}

Commodities, Crescimento económico, Desindustrialização, Extrativismo, Modelos logarítmicos. 


\section{INTRODUCCIÓN}

Aunque el modelo de desarrollo basado en la explotación de recursos naturales ha sido denominado de diversas maneras por la literatura especializada, un punto en común yace en el cuestionamiento de los aparentes beneficios para la sociedad y la economía. Beneficios que son proclamados por parte de empresas del sector minero-energético y gobiernos, que han encontrado en la promoción de este sector una fuente importante de rentas fiscales, a pesar de comprometer gravemente la base natural de aquellos territorios expuestos a extracciones masivas de sus recursos. El término "extractivismo" puede referirse a toda apropiación de recursos naturales en grandes volúmenes o bajo prácticas de alta intensidad, con fines de exportación hacia el resto del mundo [1], esto incluiría a sectores tan diversos como la minería o la industria forestal, pasando por el sector agroindustrial hasta los proyectos de infraestructura.

Si bien el grado de afectación sobre la base natural varía entre una y otra actividad, unas transforman el ambiente hasta cierto límite, ya que no se remueven elementos del entorno, pero otras lo alteran sobrepasando umbrales ecológicos, al extraer recursos por medios que implican mayores impactos sobre el entorno. A este último tipo de actividades hace referencia el presente artículo, por lo tanto, cuando se mencione al modelo extractivista, se estará aludiendo al modo de producción basado en la extracción masiva de hidrocarburos y minerales, tales como petróleo, carbón, ferroníquel y metales preciosos como el oro.

La década de los noventa, marca un punto de inflexión en la dinámica productiva colombiana, al igual que gran parte de los países de la región, donde se consolida el protagonismo de la extracción de minerales, y especialmente, la explotación de hidrocarburos. La apertura económica fue uno de esos factores externos que suscitó dicho escenario, según Svampa y Slipak (2015) obedeciendo al cumplimiento de compromisos pactados en el marco del Consenso de Washington, el cual derivó en lo que denominan Consenso de los Commodities o productos básicos, caracterizado por combinar rentabilidad extraordinaria, reprimarización de la economía y conflictos ambientales, argumentando que la actual dinámica económica basada en la demanda internacional de commodities es, al mismo tiempo, imparable y también benéfica, para el conjunto de los países latinoamericanos [2].
La especialización de los países en desarrollo en bienes primarios o básicos y su dependencia de bienes industrializados ofrecidos en su mayoría por países desarrollados, nec esarios para mantener la producción y el empleo, crea sin duda una relación asimétrica entre estos países, lo que Raúl Prebisch llamó el "intercambio desigual", expresado en la tendencia a largo plazo de la caída de los términos de intercambio y generan la extracción de valor desde la periferia a los centros [3]. Ésta especialización en el sector extractivo, está fundamentada en un postulado de la escuela clásica de pensamiento económico: las ventajas comparativas, desarrollado por David Ricardo (1772-1823), que constituye el argumento a favor del libre comercio entre las naciones. Bajo esta doctrina, un país debe especializarse en los sectores que usan recursos que abundan en sus territorios, correspondiendo ello a una estrategia 'gana-gana' para todos los que participan en el comercio.

Es así como en nombre de las "ventajas comparativas" se promueve la especialización de las economías latinoamericanas en productos primarios, lo cual produce un efecto escala que incrementa la cantidad de recursos naturales utilizados por la actividad económica, y con ello, la rematerialización de la economía mundial. Buena parte de estos recursos naturales, son generados por las economías del sur ${ }^{4}$, las cuales incrementan la exportación y explotación de sus recursos ambientales para soportar las relaciones de intercambio desiguales y el pago de sus compromisos financieros internacionales, incrementando con ello el efecto escala sobre su espacio biofísico [4]. Esto podría ayudar a explicar por qué en Colombia, desde la década de los noventa, se ha acentuado ese proceso de especialización hacia el sector minero-energético, impulsado además por las expectativas crecientes en la demanda internacional de commodities por parte de las potencias económicas emergentes: Brasil, Rusia, India y China, así como la firma de Tratados de Libre Comercio (TLC) con potencias económicas, consolidadas y emergentes [5].

El cambio estructural en el aparato productivo colombiano también puede identificarse cuando se analiza

4 El término "economías del sur" hace alusión a aquellos países suramericanos, con economías incipientes o en vía de desarrollo. El concepto está vinculado a la teoría centroperiferia promovida por Raúl Prebisch, en la que aborda el carácter asimétrico del desarrollo económico internacional. 
la balanza comercial. La figura 1 presenta el origen de los productos exportables según su participaevolución quinquenal desde 1970 hasta 2014. Puede observarse que durante la década de los setenta, según registros del Departamento Administrativo Nacional de Estadística (DANE), los productos de origen agropecuario representaban la mayor participación en la canasta exportable, con un $70 \%$ aproximadamente, siendo el café el primer renglón en el total de exportaciones con más del 60\%; paulatinamente su participación se fue reduciendo en las siguientes décadas: alcanzó a finales de los ochenta promedios de participación del $40 \%$; en los noventa descendió a $20 \%$; para quedar relegados estos productos a un escaso diez por ciento del valor total exportado. De otro lado, se observa la poca dinámica en los productos de origen industrial en participaciones alrededor del $20 \%$ y $30 \%$, alcanzando su máximo en el año 2001 , con un $43 \%$ en el total de exportaciones, reduciéndose en la actualidad al $20 \%$, mostrando la dinámica desindustrializadora del ción en el total de exportaciones,

país. Mientras que un comportamiento contrario presentó el sector minero-energético, cuya participación en el total de exportaciones aumentó 7 veces, pasando de un escaso $10 \%$ en los años setenta a un categórico 70\% en el año 2014.

En términos económicos, las investigaciones relacionadas con las implicaciones del extractivismo se concentran, en general, hacia su efecto en el crecimiento económico como variable de interés. Desde comienzos de la década de los ochenta se publicaron los estudios pioneros que abordaron la relación entre dependencia de industrias extractivas (minería e hidrocarburos) y el desempeño económico de países en vía de desarrollo. Algunos autores, como Sachs y Warner que siguieron ésta línea de investigación, detectaron una de las principales consecuencias de las economías soportadas en la exportación de recursos naturales o materias primas, la denominada "enfermedad holandesa", la cual implica la coexistencia de un boom en el comercio de bienes de un sector, y el declive o rezago de otros sectores debido a un proceso

Figura 1. Productos exportables colombianos según su origen Participación quinquenal 1970-2014.

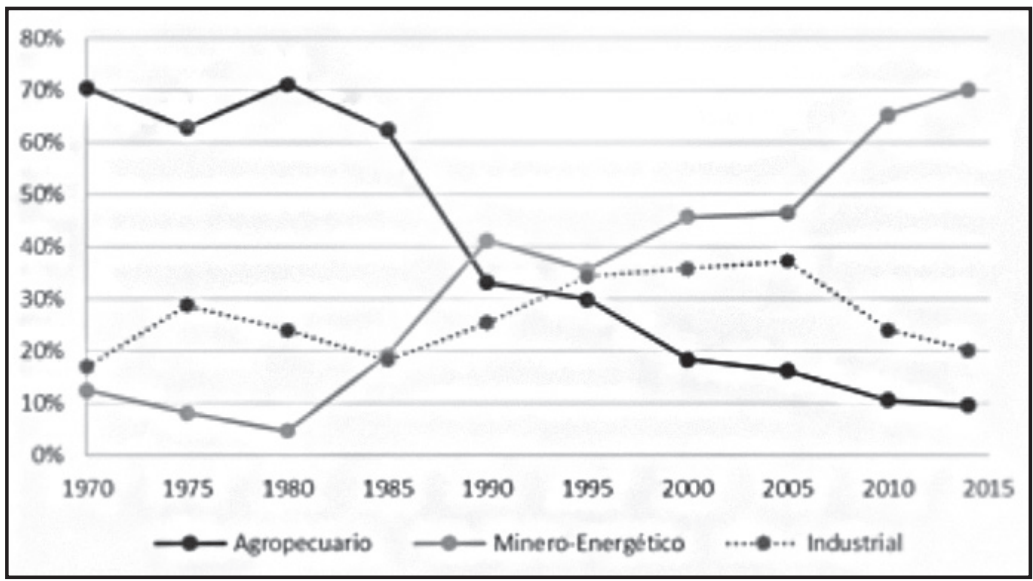

Fuente. Elaboración propia a partir de DANE (2015). de apreciación adicional de la tasa de cambio real [6]. Esta relación se utilizó para explicar el proceso de desindustrialización (contracción del sector agrícola y el industrial) de diversas economías dependientes de bienes primarios, no solamente de bienes derivados de actividades extractivas. Otros estudios mostraron evidencias sobre el lento crecimiento en aquellos países con abundancia de recursos naturales durante la década de los setenta y ochenta, respaldando la hipótesis de la paradoja "la maldición de los recursos", esto se comprobó al comparar un indicador de abundancia de recursos naturales con la Inversión Extranjera Directa, al hallar una correlación negativa [7].

Este marco histórico y analítico motivó la presente investigación, cuyo principal objetivo es identificar el aporte económico de este modo de apropiación de los recursos naturales (no renovables) al sistema productivo colombiano, así como los posibles determinantes de la dinámica exportadora extractiva. Se espera que los resultados logren aportar elementos al debate académico y político sobre la pertinencia de mantener esfuerzos e incentivos hacia el sector extractivo.

Para tal fin, el artículo se ha dividido en cuatro secciones. La primera está constituida por la presente introducción; la segunda explica el método implementado que permitió alcanzar los objetivos de la investigación, el cual está relacionado con técnicas econométricas aplicadas a series de tiempo de variables macroeconómicas, recopiladas para un horizonte temporal de 25 años; la tercera devela los resultados obtenidos a partir de la modelación econométrica, sobre la 
desvinculación del sector extractivo del sistema económico colombiano, haciendo énfasis en el escaso aporte al crecimiento económico y los posibles determinantes de las exportaciones de minerales e hidrocarburos; por último, se presentan algunas conclusiones sobre los principales hallazgos de ésta investigación.

\section{MÉTODO}

La intención es evidenciar a través de dos modelos logarítmicos el grado de asociación entre variables macroeconómicas relacionadas con el sector de minas y canteras y el crecimiento económico colombiano. Las series de tiempo que alimentaron los modelos fueron extraídas de las bases de datos abiertas que dispone el DANE, en un horizonte temporal comprendido entre 1990 y 2014, años que marcaron el antes y el después del boom extractivista en el país.

En este orden de consideraciones, el primer modelo propuesto es el siguiente:

$$
\begin{aligned}
& \operatorname{LPIBT}_{t}=\beta_{1}+\beta_{2} \text { LIEDEX }_{t}+\beta_{3} \text { LIPPIE }_{t}+ \\
& \beta_{4} \operatorname{LPIBEX}_{t}+\beta_{5} M A(q)_{t}+\omega_{t}
\end{aligned}
$$

LPIBT es el PIB total colombiano; LIEDEX hace alusión a la inversión extranjera directa al sector minero-energético. De otro lado, LIPPIE referencia el índice ponderado de precios internacionales de los principales productos exportados del sector extractivo (petróleo, carbón, níquel, oro y plata). La variable LPIBEX es el PIB extractivo del país. Para dar cuenta de eventuales choques aleatorios exógenos se introduce un promedio móvil MA(q). Finalmente es el término aleatorio de error, el cual se supone bien comportado. Vale aclarar que la letra inicial $L$ en todas ellas indica que se encuentran en expresadas en logaritmos naturales (por tanto los parámetros se deben entender como elasticidades ${ }^{5}$ ) y además se encuentran expresadas en dólares a precios constantes del año 2005.

5 El coeficiente de la elasticidad, en la notación de cálculo, se define como $(d Y / Y) /(d X / X)=[(d Y / d X) /(X / Y)]$. Por tanto, $\beta_{2}, \beta_{3}$ y $\beta_{4}$ son, en efecto, coeficientes de la elasticidad [8]. Así, los parámetros que acompañan las variables explicativas miden la elasticidad del PIBT con respecto a la IEDEX, al IPPIE y al PIBEX, en términos generales mide el cambio porcentual en $Y$ ante un pequeño cambio porcentual en $\mathrm{X}$ dado.
Lo que se espera de los parámetros es que todos sean positivos, ya que todas las variables explicativas impulsarían el PIB colombiano, incluyendo los precios internacionales de productos extractivos exportados, aunque se desconoce a priori si la relación de causalidad será inelástica, elástica o unitaria.

De otro lado, se propone un segundo modelo que permita recoger la relación entre las exportaciones del sector extractivo con el índice de precios internacionales de las exportaciones y el PIB de las economías emergentes, el cual se especifica así:

$$
\begin{aligned}
& \operatorname{LXEX}_{t}=\mathrm{a}_{1}+\mathrm{a}_{2} \text { LIPPIE }+\mathrm{a}_{3} \text { LPIBEMER }_{t}+\text { (Ec. 2) } \\
& \mathrm{a}_{4} M A(q)_{t}+\varepsilon_{t}
\end{aligned}
$$

Donde LXEX son las exportaciones del sector extractivo del país, LIPPIE es el índice ponderado de precios internacionales de los principales productos exportados del sector extractivo (petróleo, carbón, níquel, oro y plata); la variable LPIBEMER hace referencia al PIB de aquellas economías emergentes y en vía de desarrollo, principales demandantes de productos extractivos de origen colombiano (China, India, Panamá, Perú y Venezuela), la cual fue construida bajo el método de componentes principales. Para recoger posibles choques aleatorios exógenos también se introduce un promedio móvil MA(q). Finalmente denota los errores aleatorios, los cuales se suponen bien comportados. Como en el caso anterior, las variables están precedidas de la letra $L$ indicando que están expresadas en logaritmos naturales, es decir, sus parámetros son elasticidades y además, se encuentran expresadas en dólares a precios constantes del año 2005.

Previo a la estimación de los modelos se hace necesario determinar si las variables incluidas en ambas modelaciones están cointegradas o no para evitar el problema de regresiones espurias. A continuación se presentan los resultados.

\section{RESULTADOS}

\section{Análisis de cointegración}

Para examinar el orden de integración de las variables, se implementó la prueba de raíces unitarias de Dickey-Fuller Aumentada (DFA) al igual que la KPSS 
para reforzar los resultados. Los resultados resumidos se muestran en el cuadro 1.

Para ambas pruebas se encuentra que todas las variables incluidas en ambos modelos son integradas de orden uno I (1), es decir requieren de una diferenciación para ser estacionarias. Este resultado permite implementar un análisis de cointegración toda vez que las series presentan el mismo grado de integración. Por tal razón se utilizará el Test de Johansen en ambos modelos propuestos bajo el método de la traza y del máximo valor propio. Los resultados por el método de la traza se muestran en el cuadro 2 y 3.

En el cuadro 2 se muestra que existen al menos tres vectores de cointegración para el caso del modelo 1 , y en el cuadro 3, que existe al menos un vector para el caso del modelo 2. Esto permite afirmar que los modelos cointegrantes planteados en las ecuaciones 1 y 2 no son espurios o falsos. Siguiendo a Greene (2003):

"Si ambas series son I(1), entonces la diferencia parcial entre ellas debería ser estable alrededor de la media ajustada. Lo cual implica que las series fluctúan aproximadamente a la misma tasa. Las dos series que satisfacen ésta condición quiere decir que están cointegradas" [9].

En otras palabras, las variables por separado muestran comportamientos aleatorios, pero de forma agrupada establecen una combinación lineal estacionaria, que fortalece la relación a largo plazo entre las variables consideradas en ambos modelos. En este orden de ideas se pueden estimar ambos modelos sin caer en el problema de regresiones espurias.

\section{Modelación logarítmica}

Los resultados del modelo 1 se muestran en el Cuadro 4.

En este caso el modelo presenta un buen ajuste a nivel individual y conjunto, ya que los $\mathrm{p}$-valores son todos cercanos a cero y $\mathrm{R}^{2}$ es elevado $(97,9 \%)$, adicionalmente, el $\mathrm{p}$-valor del $\mathrm{F}$ estadístico es cero; frente a los signos esperados todos son correctos. En términos particulares bajo condiciones ceteris paribus, la inversión extranjera directa al sector minero energético guarda una relación inelástica frente al PIB total nacional $(0,03)$, aunque llama la atención su bajo valor, el cual es cercano a cero. De otro lado, el índice de precios internacionales de productos extractivos
Cuadro 1. Ordenes de Integración. Pruebas Dickey Fuller y KPSS.

\begin{tabular}{|l|c|c|c|c|}
\hline \multirow{2}{*}{ Variable } & \multicolumn{4}{|c|}{ Primera Diferencia } \\
\cline { 2 - 5 } & ADF & p-valor & KPSS & p-valor \\
\hline LPIBT & $-2,873$ & 0,064 & 0,179 & 0,135 \\
\hline LIEDEX & $-4,858$ & 0,001 & 0,374 & 0,184 \\
\hline LIPPIE & $-4,979$ & 0,001 & 0,200 & 0,257 \\
\hline LPIBEX & $-3,516$ & 0,017 & 0,107 & 0,153 \\
\hline LXEX & $-4,467$ & 0,002 & 0,235 & 0,180 \\
\hline LPIBEMER & $-5,479$ & 0,000 & 0,207 & 0,253 \\
\hline
\end{tabular}

Cuadro 2. Test de Cointegración de Johansen (traza). Modelo 1.

\begin{tabular}{|c|c|c|c|c|}
\hline $\begin{array}{c}\text { No. de } \\
\text { EC(s) }\end{array}$ & Eigen-value & Estadístico & $\begin{array}{c}\text { Valor } \\
\text { Crítico }\end{array}$ & Prob. $^{* *}$ \\
\hline Ninguna* & 0,726 & 77,792 & 55,246 & 0,000 \\
\hline Más de 1* & 0,658 & 49,279 & 35,011 & 0,001 \\
\hline Más de 2* & 0,543 & 25,682 & 18,398 & 0,004 \\
\hline Más de 3* & 0,319 & 8,441 & 3,841 & 0,004 \\
\hline
\end{tabular}

Cuadro 3. Test de Cointegración de Johansen (traza). Modelo 2.

\begin{tabular}{|c|c|c|c|c|}
\hline $\begin{array}{l}\text { No. de } \\
\text { EC(s) }\end{array}$ & Eigen-value & Estadístico & $\begin{array}{l}\text { Valor } \\
\text { Crítico }\end{array}$ & Prob.** \\
\hline Ninguna * & 0,969 & 90,445 & 35,011 & 0,000 \\
\hline Más de $1^{*}$ & 0,585 & 21,169 & 18,398 & 0,020 \\
\hline Más de $2 *$ & 0,163 & 3,567 & 3,841 & 0,059 \\
\hline
\end{tabular}

Cuadro 4. Estimación Modelo 1.

\begin{tabular}{|c|c|c|c|}
\hline Variable & Coeficiente & Desv. Estándar & P-valor \\
\hline C & 7,25 & 0,74 & 0,00 \\
\hline LIEDEX & 0,03 & 0,01 & 0,03 \\
\hline LIPPIE & 0,09 & 0,03 & 0,00 \\
\hline LPIBEX & 0,48 & 0,08 & 0,00 \\
\hline MA(1) & 0,71 & 0,13 & 0,00 \\
\hline R2 & 0,979 & P-Valor F & 0,000 \\
\hline
\end{tabular}

Fuente. Cálculos propios. Estimaciones bajo consistencia Newey-West.

también se comporta de forma inelástica frente al PIB total $(0,09)$ y su relación también es cercana a cero. EI PIB extractivo sigue la tendencia de las dos anteriores variables, ya que la relación es inelástica, pero muestra una relación cercana a 0,5. Finalmente los choques aleatorios previos (un rezago) existen y afectan de forma positiva al PIB total contemporáneo. En 
general, se puede concluir con todos estos resultados que el nivel de actividad económica nacional guarda una relación muy débil con el comportamiento del sector minero energético en los últimos 25 años.

En el caso de la segunda modelación, el resultado de la estimación se muestra en el cuadro 5

Como en el caso anterior, el modelo presenta una alta significancia estadística tanto a nivel individual como colectivo, pues los $p$-valores de la prueba t-student y F son todos cercanos a cero; los signos son correctos y la prueba de bondad de ajuste $\left(\mathrm{R}^{2}\right)$ es elevado (97,3\%). Específicamente hablando bajo condiciones ceteris paribus, el índice de precios internacionales guarda una relación inelástica frente a las exportaciones del sector extractivo $(0,37)$; en cuanto al PIB de los países emergentes y en vía de desarrollo la relación también es inelástica $(0,16)$. En cuanto a los choques aleatorios exógenos también afectan al PIB actual de forma rezagada. En general, se observa que para este caso, la incidencia de las economías emergentes eleva de forma positiva, aunque sutil, la exportación de productos minero-energéticos en Colombia ${ }^{6}$.

El bajo valor de los parámetros, en ambos escenarios, podría deberse al poco peso que tienen las variables analizadas en el PIB nacional. Por tal razón, se estimaron ambos modelos con las variables normalizadas para que todas tengan media cero y varianza unitaria, y así, evitar el problema de la baja participación relativa, pero los resultados siguen siendo con-

6 Finalmente, los residuales estimados de ambos modelos son estacionarios de acuerdo a la prueba ADF, aunque de nuevo este resultado no debe causar sorpresa pues las series de este modelo están cointegradas con el mismo orden.

Cuadro 5. Estimación Modelo 2.

\begin{tabular}{|c|c|c|c|}
\hline Variable & Coeficiente & Desv. Estándar & P-valor \\
\hline C & 1,591 & 0,381 & 0,000 \\
\hline LIPPIE & 0,371 & 0,198 & 0,076 \\
\hline LPIBEMER & 0,164 & 0,028 & 0,000 \\
\hline MA(1) & $-0,694$ & 0,214 & 0,004 \\
\hline R2 & 0,973 & P-Valor F & 0,000 \\
\hline
\end{tabular}

Fuente: Cálculos propios. Estimaciones bajo consistencia Newey-West cordantes con los establecidos anteriormente: todas las relaciones son inelásticas.

\section{CONCLUSIONES}

Los resultados de la investigación constituyen argumentos contundentes sobre las implicaciones para la economía que ha traído el modelo de desarrollo basado en la extracción de recursos naturales, específicamente la especialización de la economía colombiana en el sector minero-energético. Inicialmente se logró contextualizar al lector en el debate contemporáneo alrededor del extractivismo, donde además se explicaron algunos factores que han sido determinantes en la adopción del mismo, entre ellos las relaciones de dependencia a nivel mundial, en la cual el desgaste ambiental lo ponen los países del sur, que han abierto sus puertas a capitales foráneos interesados exclusivamente en el sector minero-energético.

A partir de la modelación econométrica, se encontró que Colombia a pesar de haberle apostado a un aparato productivo especializado en actividades extractivas, ha generado resultados contrarios a los esperados, donde el boom en los precios internacionales de los minerales y combustibles, a pesar de haber dinamizado el sector de minas y canteras, no ha logrado permear a la actividad económica en su conjunto. Se demostró a partir del modelo 1, que las variables del sector extractivo, como la inversión extranjera directa hacia el sector de minas y energía, los precios internacionales de los minerales e hidrocarburos, el PIB y las exportaciones extractivas, no tienen mayor peso en la explicación del PIB total colombiano. En general, se puede concluir que el nivel de actividad económica nacional ha guardado una relación muy débil con el comportamiento del sector minero-energético, en los últimos 25 años.

Se validan aquellos argumentos que califican al sector extractivo como una economía de enclave [10], en las que no ejercen los tan indispensables mecanismos de propagación del empleo y la diversificación productiva, con escasas relaciones y vinculaciones con el resto de la economía nacional. Esto se debe a que buena parte de sus insumos y tecnologías son importados, una proporción significativa de su personal técnico también es extranjero, y a su vez, no nutren cadenas industriales nacionales, sino que las exportan. Esto sumado a que la gran minería utiliza intensivamente capital que 
desplaza el enganche de mano de obra, hace que las contribuciones a las economías locales o regionales sean muy limitadas, y el grueso de sus ganancias quede en los países de origen del capital.

El modelo 2 permitió además, confirmar la relación directa entre las exportaciones de productos de origen extractivo y el índice de precios internacionales de minerales e hidrocarburos, así como el comportamiento del PIB de economías emergentes y en vías de desarrollo, no obstante se esperaba a priori que la relación fuera más estrecha. Esto teniendo en cuenta que la firma de Tratados de Libre Comercio con potencias económicas, consolidadas y emergentes, se creía que podría estar dándole un aire adicional a las exportaciones de productos básicos. El caso más sorprendente se da con India que presentó la mayor tasa de crecimiento en la demanda de minerales e hidrocarburos colombianos, según el DANE, entre 2005 y 2006 incrementó en casi 400\%. Así mismo, se esperaba que el comportamiento del índice de precios internacionales de la canasta minero-energética tuviera una mayor incidencia en la dinámica exportadora de productos extractivos nacionales.

Estos hallazgos darían cuenta del impacto que pudo tener la caída en el precio de éstas commodities a partir de 2012 en los mercados mundiales, cuyas razones son diversas; por un lado, se puede aducir una tendencia hacia la sobreproducción por parte de los principales países exportadores, aunque también disminuyó su demanda mundial en la medida en que los países lograron estabilizar sus economías. Lo anterior se puede explicar con el caso del oro, que es un depósito de valor, principalmente porque este metal es refugio para aquellos ahorradores expuestos a la incertidumbre financiera, especialmente europeos y estadounidenses fuertemente golpeados por la más reciente crisis económica mundial, contrario a otras inversiones, ya que no está ligado a la solvencia de un emisor o la estabilidad de un sistema financiero.

Resulta cuestionable sacrificar el sustrato natural con el fin de atender demandas especulativas de recursos agotables, más aún cuando no están logrando fortalecer el aparato productivo del país. Este artículo pudo corroborar que en realidad los supuestos beneficios económicos, que aparentemente permitirían saldar la creciente deuda social y ecológica del extractivismo, no se han materializado, lo cual no justifica seguir promoviendo al sector de minas e hi- drocarburos como base del crecimiento y desarrollo económico de Colombia.

Se sugiere un viraje en el modelo de desarrollo dominante, en el que se promuevan aquellas ramas de actividad económica castigadas durante más de dos décadas de hegemonía del sector de minas y energía en el país, tales como la agricultura y la industria, potencialmente menos agresivas contra el medio ambiente e importantes focos de demanda laboral.

\section{REFERENCIAS}

[1] GUDYNAS, E. et al. Teología de los extractivismos. Tabula Rasa, 24, 2016, p. 11-23.

[2] SVAMPA, M. y SLIPAK, A. China en América Latina: Del consenso de los commodities al consenso de Beijing. Revista Ensambles, 3, 2015, p. 34-63.

[3] ACOSTA, A. Post-extractivismo: entre el discurso y la praxis. Algunas reflexiones gruesas para la acción. Ciencia Política, 11(21), 2016, p. 287-332.

[4] PÉREZ-RINCÓN, M. En: Minería en Colombia: Control público, memoria y justicia socio-ecológica, movimientos sociales y posconflicto. Conflictos ambientales en Colombia: Inventario, caracterización y análisis. 1 ed. Bogotá (Colombia): Contraloría General de la República, 2014, p. 253-318.

[5] SARMIENTO-CASTILLO, J. y PÉREZ-RINCÓN, M. Caracterización del proceso de especialización de la economía colombiana hacia el sector extractivo. Ambiente y Sostenibilidad, 5, 2015, p. 118-130.

[6] GODA, T.y TORRES, A. Flujos de capital, recursos naturales y enfermedad holandesa: el caso colombiano. Ensayos Sobre Política Económica, 33(78), 2015, p. 197-206.

[7] CAMPO, J. y SANABRIA, W.A. Recursos Naturales y Crecimiento Económico en Colombia: ¿Maldición de los Recursos?. Perfil de Coyuntura Económica, 21, 2013, p. 17-37.

[8] GUJARATI, D. y PORTER, D. Econometría. 5 ed. México D.F. (México): McGraw-Hill, 2010, 921 p.

[9] GREENE, W. Econometric Analysis. 5 ed. New Jersey (USA): Prentice Hall, 2003, 959 p.

[10] ESPITIA, J.E. En: Minería en Colombia: Daños ecológicos y socio-económicos y consideraciones sobre un modelo minero alternativo. La distribución regional de la actividad agrícola, minera y no minera en Colombia 1975-2012. 1 ed. Bogotá (Colombia): Contraloría General de la República, 2014, p. 381-437. 